PRAMANA

(C) Indian Academy of Sciences

Vol. 78 , No. 6

— journal of

June 2012

physics

p. 981

\title{
Erratum to: Measurement of peak fluence of neutron beams using Bi-fission detectors
}

\author{
R K JAIN ${ }^{1}$, ASHOK KUMAR ${ }^{2, *}, \mathrm{~N} \mathrm{~L} \mathrm{SINGH}^{3}, \mathrm{~L}_{\text {TOMMASINO}}^{4}$ \\ and $\mathrm{B}$ K SINGH ${ }^{5}$ \\ ${ }^{1}$ Department of Physics, School of Basic \& Applied Sciences, Shobhit University, \\ Meerut 250 110, India \\ ${ }^{2}$ Department of Applied Science (Physics), Vidya College of Engineering, Meerut 250 010, India \\ ${ }^{3}$ Physics Department, M.S. University of Baroda, Vadodara 390 002, India \\ ${ }^{4}$ National Agency for Environment Protection and Technical Services, APAT, via V. Brancati-48, \\ 00144, Rome, Italy \\ ${ }^{5}$ High Energy Physics Laboratory, Department of Physics, Banaras Hindu University, \\ Varanasi 221 005, India \\ *Corresponding author. E-mail: ashokblp@gmail.com
}

(Pramana - J. Phys. 78, 493-498 (2012))

The original publication of this article (10.1007/s12043-011-0236-7), unfortunately bears incorrect information in table 1 . The corrected table 1 is shown below.

Table 1. Measured and calculated neutron fluence.

\begin{tabular}{lcccc}
\hline $\begin{array}{c}\text { Energy } \\
(\mathrm{MeV})\end{array}$ & $\begin{array}{c}\text { (Sparks/neutrons)* } \\
10^{-7}\end{array}$ & Sparks $/ \mathrm{cm}^{2}$ & $\begin{array}{c}\text { Measured neutron } \\
\text { peak fluence }\end{array}$ & $\begin{array}{c}\text { Calculated neutron } \\
\text { peak fluence }\end{array}$ \\
\hline 100 & $2.2 \pm 0.2$ & $16 \pm 2$ & $(7.7 \pm 0.4) * 10^{7}$ & $(7.3 \pm 0.8) * 10^{7}$ \\
\hline
\end{tabular}

